\title{
Examination of a region showing linkage map discrepancies across sheep breeds
}

\author{
Allan F. McRae, ${ }^{\star}$ Dario Beraldi \\ Institute of Evolutionary Biology, University of Edinburgh, Edinburgh, EH9 3JT, United Kingdom \\ Received: 28 June 2005 / Accepted: 11 January 2006
}

\begin{abstract}
The availability of accurate linkage maps is an important step for the localization of genetic variants of interest. However, most studies in livestock assume the published map is applicable in their population despite the large differences between the breeds of a species. A region of sheep Chromosome 1 was previously identified as providing evidence for a marker order inconsistent with the published linkage map. In this study the identified region was investigated in more detail. Four microsatellite markers covering the central $5 \mathrm{cM}$ of the inconsistent region and two flanking markers were genotyped in three sheep breeds, a commercial population (Charollais), an experimental population (Scottish Blackface), and a feral population (Soay). With the inclusion of the published linkage map, this provided evidence for three different marker orders across four sheep populations. Evidence for selection in this region was investigated using both a single-point allelic competition model and a multipoint allele-sharing statistic. Only the Charollais population provided evidence for selection, with significant transmission bias observed at marker BM7145. The implications of variation in linkage maps on the design and analysis of fine-mapping studies are discussed.
\end{abstract}

\section{Introduction}

The advent of molecular markers has seen the construction of linkage maps for a variety of commercially important livestock species including

\footnotetext{
^Present address: Genetic Epidemiology Group, Queensland Institute of Medical Research, Post Office, Royal Brisbane Hospital, Brisbane, 4006, Australia

Correspondence to: Allan F. McRae; E-mail: allan.mcrae@qimr. edu.au
}

cattle (Barendse et al. 1997; Kappes et al. 1997; Ihara et al. 2004), chicken (Groenen et al. 2000; Schmid et al. 2000), deer (Slate et al. 2002), pig (Archibald et al. 1995; Rohrer et al. 1996), and sheep (de Gortari et al. 1998; Maddox et al. 2001). The primary purpose of such linkage maps is to provide a framework to locate genes of commercial importance, either through linkage mapping or comparative analysis. In a typical linkage study, the published linkage map is used, rather than constructing separate linkage maps for each population studied, because this allows for comparisons across studies and removes the need to publish similar linkage maps for each population studied. This approach of using published linkage maps is also justifiable given the more comprehensive coverage of the genome in the mapping populations.

The use of linkage maps in breeds of animals other than those that were used in the construction of the published map requires recognition of the limitations of the methods used in their construction. The map distances provided depend on the mapping function used and are an average over the pedigree(s) investigated, thus averaging any variation between individuals. As some of the variation in recombination has been shown to be heritable (Kong et al. 2004), it is likely that differences in map distances exist between populations. Such differences have been observed between ethnic groups in human populations (Jorgenson et al. 2005). However, differences between the actual map distances and those used in linkage analysis have little effect in practice (Dodds et al. 2004) and are difficult to detect statistically.

The map order is fundamentally more important in gene mapping, especially when trying to physically locate the underlying polymorphism controlling the trait of interest. As such, the map order is generally the primary focus of linkage map con- 
struction with map distances being estimated only as a secondary consequence. This is evident in the published maps that, in general, provide a framework map where the likelihood of the order given is greater than the likelihood of any other order by at least some predefined threshold, but provide no standard errors on distances. A typical threshold of 3 LOD (logarithm of the odds) is based on a recommendation given by Morton (1955) for a correction for the multiple testing involved in linkage analysis. Given that the latest linkage maps for the major livestock species now contain more than 1000 loci, a situation clearly not considered by Morton, the continued use of this threshold is likely to result in some errors in the estimated marker order.

Despite these limitations in linkage map construction, differences in linkage map order have not been forthcoming. However, a large number of chromosomal rearrangements have been detected in individual animals and, in some cases, their immediate relatives, using chromosome staining in pigs (Henricson and Bäckström 1964; Popescu and Boscher 1986; Ducos et al. 2002), cattle (Christensen et al. 1992; Pinton et al. 1997; Joerg et al. 2001), and chickens (Ramos et al. 1999). The primary reason why chromosomal abnormalities are not detected in linkage studies is the associated reduction in fertility of carriers (Gustavsson 1980). This can be mediated by prezygotic mechanisms such as an increase in nondisjunction of chromosomes or chromosome segments at meiosis that result in gametes with abnormal cytology and by a reduction in sperm fertility through decreased motility and abnormal morphology (Guttenbach et al. 1997), or by postzygotic mechanisms including an increase in spontaneous abortions of fetuses (Kalousek and Lau 1992). During meiosis, selection against a chromosomal inversion can be reduced through the formation of a loop structure during the first metaphase (McClintock 1931, 1933) or the suppression of recombination in the region (e.g., Martin et al. 1994; Brown et al. 1998). Such suppression due to the presence of a small inversion has previously been demonstrated to be the cause of a fine-scale difference in recombination rate between bulls (Park et al. 1995, 1999). It follows that selection against an inversion may occur primarily at a postzygotic level. This may be detected as the unequal transmission of alleles in the inverted segment of a heterozygous animal.

Recently, McRae et al. (2005) reported the detection of an inconsistency between the published sheep linkage map (Maddox et al. 2001) and the linkage map in a commercial population of Charollais sheep. When compared with the published linkage map, markers MCM137 and BM6506 on Chromosome 1 were in an inverted order relative to the flanking markers BMS527 and BM8246. This article examines this region with more detail in the Charollais population with the genotyping of two further markers. The region is also examined in an experimental sheep population and a feral sheep population.

\section{Materials and methods}

Animals. Three sheep breeds were examined at the markers of interest. One of theses breeds, the Charollais, was studied through sire referencing schemes in commercial populations from the United Kingdom. The second, the Scottish Blackface, was an experimental sheep population at Roslin Institute. The third breed examined was a feral Soay sheep population from St. Kilda, Scotland [see Chapter 2 of Clutton-Brock and Pemberton (2004) for a description of this population]. The Charollais sheep pedigree has been described elsewhere (McRae et al. 2005). Briefly, a complex pedigree of 570 animals derived from the descendants of five widely used sires was selected for a QTL mapping study. Of the 570 animals, 420, mainly descendants, were genotyped. The Scottish Blackface sheep pedigrees consisted of nine half-sib families of sizes ranging from 11 to 145, averaging 72 sheep. The Soay sheep pedigree was selected from a larger pedigree constructed using molecular techniques with the aim of maximizing power for QTL detection. First, halfsibships of 12 or more animals and their common parent were selected followed by the addition of halfsibships of at least 10 animals that were linked to previously selected animals. This selected 588 animals for genotyping. The pedigree was extended further by including all available ancestral information for the selected individuals resulting in a total pedigree size of 868 animals.

Genotyping. The region of Chromosome 1 showing a marker order inconsistency in the Charollais sheep population was further investigated with the genotyping of two additional microsatellite markers, BM7145 and BMS4008. The markers BM6506 and MCM137, which originally indicated the marker order inconsistency, were also regenotyped to verify the accuracy of the commercial genotyping service. All four of these markers, as well as two flanking markers from the Charollais sheep map, BM8246 and BMS527, were genotyped in the Scottish Blackface and Soay sheep pedigrees. Additional flanking markers (DB6, TGLA415, and SOX2) were genotyped in the Scottish Blackface pedigree to 
increase the precision of estimation of marker order as not all families were informative for the flanking markers. This was especially true for marker BM8246 where only one family was informative. Because no significant deviation from the published map order was observed in the extra flanking markers, only the positions of the flanking markers common to all populations are reported. Microsatellite allele lengths were determined using an ABI 3730 DNA analyzer. Genotypes were automatically determined using GeneMapper followed by manual checking of the results. Genotype information is summarized in Table 1. The lower number of informative meioses in the Soay population compared with that in both the Charollais and Scottish Blackface is expected since the Soay population history has involved a number of bottlenecks (Clutton-Brock and Pemberton 2004). Based on the published map distances and the observed number of informative meioses, the resolution of marker order is theoretically achievable in all populations but is approaching the limits of resolution in the Soay pedigree.

Construction of linkage maps. Linkage maps were constructed using CRIMAP (Green et al. 1990). CRIMAP is widely used in the construction of linkage maps in livestock populations because of its ability to handle large pedigrees from outbred populations. This is achieved by the simplification of the full-likelihood model through the exclusion of individuals who are uninformative or potentially uninformative for linkage (i.e., individuals that are homozygous at a locus or those that have a missing genotype that could possibly be homozygous). This simplifies the likelihood by avoiding the summation over all possible genotype probabilities in individuals with missing data. As the information ignored is consistent across all marker orders, the likelihoods of different orders are still able to be compared.

Because potential false "double recombinant" individuals may result in a bias in linkage map construction, these need to be removed before linkage map construction. This is especially important in the central markers of the region studied because these cover only a few centiMorgans and are very unlikely to contain true double recombinants. However, the detection of such individuals requires prior knowledge of the linkage map order. This was accounted for using the following multistage procedure. Initially, the published linkage map order was assumed and individuals with potential double recombinant genotypes in the central markers were detected using the "chrompics" option of CRIMAP and removed from the pedigree. On average this re- moved five individuals from the pedigree. Then the likelihood of all permutations of nonflanking marker orders was calculated. If a more likely marker order were detected, the removed individuals were replaced in the pedigree and the process was repeated using the new marker order. Once the best marker order had been found using this method, all other orders having a log-likelihood within 3 LOD from that of the best marker order were recorded so that potential type I error could be assessed. All map likelihoods in this study were calculated using sexaveraged recombination distances, although this reduced to the male linkage map in the case of the Scottish Blackface population where recombination information was available from only male individuals. In the other populations, specifying a linkage map for each sex provided the same results (data not shown).

The current best estimate of the linkage map from the (AgResearch) International Mapping Flock (IMF) used in the construction of the published map was obtained from the framework given in Maddox et al. (2001) with distances as given on the Australian Sheep Genetics web site (Maddox 2003). Only the central markers used in this study were placed at specific points on the published framework map, but the potential positions shown for the flanking markers were both outside this region. This indicates that the IMF population has no other marker order with likelihood within 3 LOD of the order considered here.

Detection of transmission distortion. As outlined above, postzygotic selection against a chromosomal rearrangement can result in unequal transmission of chromosome types to a heterozygous individual's offspring. The detection of transmission distortion was achieved in two ways. The first approach involved an application of the Bradley-Terry model for ranking sports teams (Bradley and Terry 1952). In this case, the alleles at a marker locus are ranked according to an indicator of transmission potential and tested for deviation from equality (Sinsheimer et al. 2000). The analysis was performed using the "Gamete_competition" module of Mendel (v5.7; Lange et al. 2001). Alleles with low frequencies in the population $(>5 \%)$ were pooled to remove potential bias associated with the use of large sample results in evaluating significance. Because this single marker analysis potentially loses power as a result of not incorporating transmission information from multiple markers, an additional multipoint test for transmission distortion was also performed. The basis for the multipoint statistic is the observation that preferential 
Table 1. Summary of marker information in the three examined populations

\begin{tabular}{|c|c|c|c|c|}
\hline Population & Marker & Number of alleles & $P I C^{\mathrm{a}}$ & Informative meioses \\
\hline Charollais & $\begin{array}{l}\text { BMS527 } \\
\text { MCM137 } \\
\text { BM7145 } \\
\text { BM6506 } \\
\text { BMS4008 } \\
\text { BM8246 }\end{array}$ & $\begin{array}{r}8 \\
15 \\
7 \\
6 \\
7 \\
9\end{array}$ & $\begin{array}{l}0.69 \\
0.83 \\
0.72 \\
0.70 \\
0.70 \\
0.82\end{array}$ & $\begin{array}{l}373 \\
494 \\
332 \\
308 \\
366 \\
483\end{array}$ \\
\hline Soay & $\begin{array}{l}\text { BMS527 } \\
\text { MCM137 } \\
\text { BM7145 } \\
\text { BM6506 } \\
\text { BMS4008 } \\
\text { BM8246 }\end{array}$ & $\begin{array}{l}5 \\
7 \\
4 \\
5 \\
5 \\
5\end{array}$ & $\begin{array}{l}0.42 \\
0.70 \\
0.64 \\
0.63 \\
0.74 \\
0.59\end{array}$ & $\begin{array}{l}153 \\
176 \\
112 \\
153 \\
126 \\
128\end{array}$ \\
\hline $\begin{array}{l}\text { Scottish } \\
\text { Blackface }\end{array}$ & $\begin{array}{l}\text { BMS527 } \\
\text { DB6 } \\
\text { MCM137 } \\
\text { BM7145 } \\
\text { BM6506 } \\
\text { BMS4008 } \\
\text { TGLA415 } \\
\text { SOX2 (LS6) } \\
\text { BM8246 } \\
\end{array}$ & $\begin{array}{r}7 \\
16 \\
15 \\
8 \\
6 \\
9 \\
10 \\
17 \\
8\end{array}$ & $\begin{array}{l}0.75 \\
0.78 \\
0.89 \\
0.60 \\
0.63 \\
0.73 \\
0.52 \\
0.73 \\
0.60 \\
\end{array}$ & $\begin{array}{r}328 \\
378 \\
383 \\
189 \\
343 \\
356 \\
191 \\
375 \\
37\end{array}$ \\
\hline
\end{tabular}

${ }^{\mathrm{a} P I C}=$ Polymorphic Information Content (Botstein et al. 1980).

transmission at a locus results in an increase in relatedness among an individual's offspring. Thus, an indicator of transmission distortion in a general pedigree of size $n$ can be constructed by

$\mathrm{TDI}=\sum_{i=1}^{n} \sum_{j=1}^{i} \hat{\pi}_{i j}$

where $\hat{\pi}_{i j}$ is the estimated identity-by-descent (IBD) probability for animals and $j$. Multipoint IBD values were estimated by Markov chain Monte Carlo (MCMC) sampling using the program LOKI (Heath 1997) and assuming the most likely map order in each population. Average IBD coefficients were calculated from the mean of 10,000 samples taken at every tenth iteration after a 1000-iteration burn-in. The empirical distribution of the indicator statistic was assessed by 1,000,000 gene-dropping simulations. In these simulations, all founders were as- sumed to have unique alleles because this allowed for rapid calculation of IBD values.

\section{Results}

Linkage map construction. The estimated linkage maps for the populations for the region examined are given in Table 2. In the Charollais and Soay sheep populations, the most likely map order was greater than 3 LOD more likely than any other marker order. The order of markers BMS527 and MCM137 was not able to be elucidated in the Scottish Blackface population with the most likely map order positioning these markers together. The Charollais population shows strong evidence against having the marker order given by the IMF populations $(-10.0$ LOD) and the order given by the Soay sheep population (-12.0 LOD). Similarly, the Soay sheep popu-

Table 2. Estimated marker positions for the most likely linkage map order within each population

\begin{tabular}{|c|c|c|c|c|}
\hline \multirow[b]{2}{*}{ Marker } & \multicolumn{4}{|c|}{ Population } \\
\hline & $I M F^{\mathrm{a}}$ & Charollais & Soay & Scottish Blackface \\
\hline BMS527 & $0.0^{(1)}$ & $0.0^{(1)}$ & $0.0^{(1)}$ & $0.0^{(1)}$ \\
\hline MCM137 & $18.7^{(2)}$ & $18.0^{(3)}$ & $23.0^{(3)}$ & $19.6^{(2 / 3)}$ \\
\hline BM7145 & $19.8^{(3)}$ & $18.6^{(4)}$ & $22.6^{(2)}$ & $19.6^{(2 / 3)}$ \\
\hline BM6506 & $20.9^{(4)}$ & $15.9^{(2)}$ & $25.1^{(4)}$ & $20.3^{(4)}$ \\
\hline BMS4008 & $22.0^{(5)}$ & $19.8^{(5)}$ & $26.4^{(5)}$ & $22.3^{(5)}$ \\
\hline BM8246 & $27.2^{(6)}$ & $25.6^{(6)}$ & $34.2^{(6)}$ & $29.7^{(6)}$ \\
\hline
\end{tabular}

No other orders were within 3 LOD of the most likely order. The relative position of each marker is given beside the estimates. ${ }^{\mathrm{a}}$ Marker distance is measured from BMS527, which is at position $213.9 \mathrm{cM}$ on Chromosome 1 in the published linkage map. 
Table 3. Summary of the five most likely marker orders for each of population

\begin{tabular}{lccc}
\hline Population & Order & $\log _{10}$-likelihood & Map length \\
\hline IMF & $1,2,3,4,5,6$ & - & 27.2 \\
Charollais & $1,4,2,3,5,6$ & -125.97 & 25.6 \\
& $1,4,2,5,3,6$ & -129.12 & 26.2 \\
& $1,4,3,2,5,6$ & -129.65 & 26.0 \\
Soay & $1,4,5,2,3,6$ & -129.79 & 26.8 \\
& $1,4,5,2,3,6$ & -131.72 & 26.6 \\
& $1,3,2,4,5,6$ & -124.48 & 34.2 \\
& $1,2,3,4,5,6$ & -127.63 & 34.8 \\
Scottish Blackface & $1,3,2,5,4,6$ & -130.05 & 35.0 \\
& $1,5,4,2,3,6$ & -132.99 & 40.4 \\
& $1,2,3,5,4,6$ & -133.22 & 35.6 \\
& $1,2 / 3,4,5,6$ & -168.51 & 29.7 \\
& $1,4,2 / 3,5,6$ & -171.64 & 31.1 \\
& $1,2,4,3,5,6$ & -172.59 & 30.2 \\
\hline
\end{tabular}

Locus numbers represent the order from the IMF population starting at BMS527. Markers with tied positions are indicated with a slash.

lation provides evidence against having the IMF marker order $(-3.2 \mathrm{LOD})$ and the order in the Charollais population (-12.8 LOD). The marker order obtained in the Scottish Blackface populations is consistent with the orders given in both the IMF and Soay populations but not the Charollais order $(-3.2$ LOD). A summary of the five most likely map orders in the three populations examined is given in Table 3.

Detection of transmission distortion. The significances of the single-point analysis of allele transmission are given in Table 4. Only the Charollais population shows any evidence of transmission distortion with marker BM7145 being significant at the $5 \%$ level. While this result could potentially be caused by problems with the PCR, e.g., uneven amplification of alleles, such problems were not apparent during the scoring of genotypes. Also, there was no excess in Mendelian inconsistencies at this marker relative to other markers. The multipoint transmission distortion indicator statistic provides no significant results at any of the marker positions (Table 5). However, in the Charollais population the two flanking markers show less allele-sharing than all the internal markers, a situation expected if transmission distortion is occurring due to selection against chromosomal heterogeneity in this region. Marker BMS527 in the Scottish Blackface population approaches significance in the multipoint analysis but not in the single-point analysis. However, it provides no evidence for selection on a chromosomal rearrangement because it is a flanking marker for the region of interest.

\section{Discussion}

With the increasing effort to fine-map and ultimately locate variants in regions detected to have an effect on commercially important traits in previous genome-wide scans, it is becoming increasingly important to have dense and accurate linkage maps. Here, a difference between the linkage maps of the

Table 4. Significance of single-point analysis of transmission distortion

\begin{tabular}{llcr}
\hline & \multicolumn{3}{c}{ Population } \\
\cline { 2 - 4 } Marker & Charollais & Scottish Blackface & Soay \\
\hline BMS527 & 0.924 & 0.146 & 0.469 \\
MCM137 & 0.755 & 0.361 & 0.518 \\
BM7145 & $\mathbf{0 . 0 1 5 ^ { \mathbf { a } }}$ & 0.453 & 0.625 \\
BM6506 & 0.416 & 0.157 & 0.323 \\
BMS4008 & 0.150 & 0.899 & 0.708 \\
BM8246 & 0.562 & 0.968 & 0.432 \\
\hline
\end{tabular}

${ }^{a}$ Only marker BM7145 in the Charollais sheep population shows significant amount of transmission distortion at the $5 \%$ level. 
Table 5. Multipoint analysis of transmission distortion

\begin{tabular}{llll}
\hline & & \multicolumn{1}{c}{ Population } \\
\cline { 2 - 4 } Marker position & Charollais & Scottish Blackface & Soay \\
\hline $1^{\text {(BMS527) }}$ & 0.200 & 0.050 & 0.416 \\
2 & $0.123^{\text {(BM6506) }}$ & $0.237^{(\mathrm{BM} 7145)}$ & $0.638^{(\mathrm{MCM} 137)}$ \\
3 & $0.132^{\text {(MCM137) }}$ & $0.240^{\text {(MCM137) }}$ & $0.639^{(\mathrm{BM} 7145)}$ \\
4 & $0.095^{\text {(BM7145) }}$ & $0.314^{\text {(BM6506) }}$ & $0.699^{(\mathrm{BM} 6506)}$ \\
$5^{\text {(BMS4008) }}$ & 0.078 & 0.447 & 0.671 \\
$6^{\text {(BM8246) }}$ & 0.176 & 0.264 & 0.678 \\
\hline
\end{tabular}

The proportion of gene-drop replicates having larger transmission distortion indicator values than that calculated from marker data is shown. Although not significant, the central markers in the Charollais sheep linkage map show increased allele sharing compared to the flanking markers.

IMF population and the Charollais and Soay sheep populations has been demonstrated. The map orders from the Charollais and Soay populations also differ from each other. The estimated marker order in the Scottish Blackface is consistent with the order given by both Soay and IMF populations. Thus, this study has identified a total of three different map orders across four sheep populations.

The marker orders observed in this study require at least two chromosomal rearrangements since the sheep breeds last shared a common ancestor. The most likely order in the Soay sheep population shows a two-marker inversion (MCM137 and BM7145) compared with the IMF linkage map. The relationship between the linkage map for the Charollais sheep population and that for the other populations depends on which map is chosen as the correct map. The most likely order in the Charollais population is a three-marker inversion (BM7145, MCM137, BM6506) from the order given in the Soay sheep population and requires either two inversions or a translocation to achieve the order given by the IMF population. This suggests that the most parsimonious solution to the ancestry of these sheep breeds is to consider the Soay sheep as an outgroup to the IMF and Charollais populations with a different inversion occurring in both the IMF and Charollais lines once these had split. This interpretation is consistent with the primitive status of the Soay sheep. Unfortunately, not all these markers amplify in other livestock species, or, if they do amplify, their order cannot be elucidated using the current linkage mapping populations, so it is not possible to further clarify the history of this region.

This study provides some evidence for selection occurring in the region of interest in the Charollais sheep population through the observation of unequal transmission of alleles at BM7145 and an increase in allele-sharing at the central marker positions compared with the flanking marker positions. Both methods used to detect possible transmission distortion have potential pitfalls that need to be examined. The single-point analysis ranks the alleles at a marker locus based on their transmission probabilities when in competition with each other (i.e., in a heterozygous individual). Thus, it is a test of association between marker alleles and the locus causing the transmission distortion. While it is likely that a chromosomal rearrangement occurs initially in one individual, any association with marker alleles is likely to be incomplete, thus reducing the power for detecting transmission distortion. Also, the requirement of the pooling of rare alleles for the large sample distribution of the test statistic to be applicable may potentially combine alleles at the opposite end of the transmission spectrum, biasing the results toward the null hypothesis. The multipoint test statistic has an underlying bias induced by the methodology used to estimate the null distribution. In the gene-dropping simulations, all founder individuals were considered to have unique alleles because this allowed for rapid computation of IBD values that are otherwise computationally demanding in the complex pedigrees used. A consequence of this assumption is an increased range in the simulated null distribution. It can be shown that this increase occurs primarily at the higher end of the distribution of the test statistic, biasing estimated $p$-values upward. Thus, the evidence for selection in the examined region is increased by the knowledge that marker inheritance is not fully determinable given the number of alleles at the marker loci.

While the detection of weak transmission distortion in the Charollais sheep provides indirect evidence for the presence of multiple linkage map orders in a population, the methodology used in the estimation of linkage maps assumes only a single map order. This limitation in the methodology is not readily removed, especially given the complex pedigree structure in the Charollais sheep population. The most likely map order obtained from CRIMAP represents an "average" map order within the population whose value depends on the map 
orders within the population and their relative frequencies and thus does not necessarily represent an order present in the population. However, such heterogeneity in chromosome structure is also likely to reduce the difference in likelihoods between competing map orders. The difference of 3.19 LOD between the two most likely orders in the Charollais population suggests that any effect of homogeneity on linkage map selection is relatively limited.

The observation of heterogeneity of chromosome structure has implications for the design and analysis of fine-mapping studies. Because the map cannot be assumed to be the same as the published map, information on linkage will also need to be collected. Thus, fine-mapping using linkage disequilibrium in a random sample from a population should be undertaken with caution. Instead, designs such as several large half-sib families are more appropriate because all individuals provide information through both linkage and linkage disequilibrium. The choice of analysis methodology used in fine-mapping studies also depends on the amount of information about linkage that is available. While single-locus methods such as regression of trait values on genotype do not directly require linkage information for their use, the following attempts to characterize the underlying genetic variants in the detected region may be hampered without this information. Also, given the large amount of variability in the amount of linkage disequilibrium between pairs of loci separated by a fixed distance in livestock populations (Farnir et al. 2000; McRae et al. 2002; Nsengimana et al. 2004), multilocus methods that average out this variability are likely to be more powerful than single locus methods. All multilocus methods will require a genetic map to be known accurately so the appropriate weighting of information at each locus is achieved. Incorrect specification of linkage maps may result the localization of a locus to an incorrect region.

\section{Acknowledgments}

The Charollais study was funded by the Department for Environment, Food and Rural Affairs (Defra), Scottish Executive Environment and Rural Affairs Department (SEERAD) and the Meat and Livestock Commission (MLC) through the LINK Sustainable Livestock Production Programme. The Scottish Blackface study was funded by Defra. The authors are grateful to S. Bishop, G. Walling, A. Wilson, E. Karamichou, and G. Davies for their role in the collection and extraction of DNA samples and initial data checking. This collection was helped with input from Charollais Sire Referencing Ltd., and Mr. R.
Pratt in particular, and from D. Wallace and D. McGavin at Roslin Institute's Blythbank farm. The authors also acknowledge assistance from MLC's Signet Breeding Services and Edinburgh Genetics. The collection of the data on the Soay sheep used in this study was funded by the UK Natural Environmental Research Council and the Wellcome Trust. The authors are grateful to the National Trust for Scotland, Scottish Natural Heritage, the Ministry of Defence, Eurest, and QinetiQ for permission and practical support for the St. Kilda Soay Sheep Project. The authors thank all who have contributed to the Soay sheep data set, especially J. Pemberton and J. Pilkington. They thank P. Visscher for many helpful discussions on this topic. Th article was improved by the helpful suggestions of two anonymous referees. A.M. was funded by a Commonwealth Scholarship.

\section{References}

1. Archibald AL, Haley CS, Brown JF, Couperwhite S, McQueen HA, et al. (1995) The Pigmap Consortium linkage map of the pig (Sus scrofa). Mamm Genome 6, $157-175$

2. Barendse W, Vaiman D, Kemp SI, Sugimoto $Y$, Armitage SM, et al. (1997) A medium-density genetic linkage map of the bovine genome. Mamm Genome 8, $21-28$

3. Botstein D, White RL, Skolnick M, Davis RW (1980) Construction of a genetic linkage map in man using restriction fragment length polymorphisms. Am J Hum Genet 32, 314-331

4. Bradley RA, Terry ME (1952) Rank analysis of incomplete block designed. Biometrika 39, 324-345

5. Brown GM, Leversha M, Hulten M, Ferguson-Smith MA, Affara NA, et al. (1998) Genetic analysis of meiotic recombination in humans by use of sperm typing: Reduced recombination within a heterozygous paracentric inversion of chromosome 9q32-q34.3. Am J Hum Genet 62:1484-1492

6. Christensen K, Agerholm JS, Larsen B (1992) Dairy breed bull with complex chromosome translocation: Fertility and linkage studies. Hereditas 117, 199-202

7. Clutton-Brock TH, Pemberton JM, eds (2004) Soay Sheep. Dynamics and Selection in an Island Population (Cambridge: Cambridge University Press)

8. de Gortari MJ, Freking BA, Cuthbertson RP, Kappes SM, Keele JW, et al. (1998) A second-generation linkage map of the sheep genome. Mamm Genome 9, 204-209

9. Dodds KG, Ball R, Djorovic N, Carson SD (2004) The effect of an imprecise map on interval mapping QTLs. Genet Res 84, 47-55

10. Ducos A, Pinton A, Yerle M, Séguéla A, Berland H-M, et al. (2002) Cytogenetic and molecular characterization of eight new reciprocal translocations in the pig species. Estimation of their incidence in French populations. Genet Sel Evol 34, 389-406 
11. Farnir F, Coppieters W, Arranz J-J, Berzi P, Cambisano $\mathrm{N}$, et al. (2000) Extensive genome-wide linkage disequilibrium in cattle. Genome Res 10, 220-227

12. Green P, Falls K, Crooks S (1990) Cri-Map Version 2.4 (St. Louis, MO: School of Medicine, Washington University)

13. Groenen MAM, Cheng HH, Bumstead N, Benkel BF, Briles WE, et al. (2000) A concensus linkage map of the chicken genome. Genome Res 10, 137-147

14. Gustavsson I (1980) Chromosome abberations and their influence on the reproductive performance of domestic animals-A review. Z Tierzücht Züchtungsbiol 97, 176-195

15. Guttenbach M, Engel W, Schmid M (1997) Analysis of structural and numerical chromosome abnormalities in sperm of normal men and carriers of constitutional chromosome aberrations: A review. Hum Genet 100, $1-21$

16. Heath SC (1997) Markov Chain Monte Carlo segregation and linkage analysis for oligogenic models. Am J Hum Genet 61, 748-760

17. Henricson B, Bäckström L (1964) Translocation heterozygosity in a boar. Hereditas 52, 166-170

18. Ihara N, Takasuga A, Mizoshita $\mathrm{K}$, Takeda $\mathrm{H}$, Sugimoto M, et al. (2004) A comprehensive genetic map of the cattle genome based on 3802 microsatellites. Genome Res 14, 1987-1998

19. Joerg H, Garner D, Rieder S, Suwattana D, Stranzinger G (2001) Molecular genetic characterization of Robertsonian translocations in cattle. J Anim Breed Genet $118,371-377$

20. Jorgenson E, Tang H, Gadde M, Province M, Leppert $M$, et al. (2005) Ethnicity and human genetic linkage maps. Am J Hum Genet 76, 276-290

21. Kalousek DK, Lau AE (1992) Pathology of spontaneous abortion. In Dimmick JE, Kalousek DK, eds. Developmental Pathology of the Embryo and Fetus, (Philadelphia: Lippincott), pp 55-82

22. Kappes SM, Keele JW, Stone RT, McGraw RA, Sonstegard TS, et al. (1997) A second-generation linkage map of the bovine genome. Genome Res 7, 235-249

23. Kong A, Barnard J, Gudbjartsson DF, Thorleifsson G, Jonsdottir G, et al. (2004) Recombination rate and reproductive success in humans. Nat Genet 36, 1203-1206

24. Lange K, Cantor R, Horvath S, Perola M, Sabatti C, et al. (2001) Mendel version 4.0: A complete package for the exact genetic analysis of discrete traits in pedigree and population data sets. Am J Hum Genet 69 (Suppl), A1866

25. Maddox J (2003) Australian Sheep Gene Mapping Web Site-Current sex averaged, female, male distances for best position maps (v4.2). Available at http:// rubens.its.unimelb.edu.au/ jillm/jill.htm

26. Maddox JF, Davies KP, Crawford AM, Hulme DJ, Vaiman D, et al. (2001) An enhanced linkage map of the sheep genome comprising more than 1000 loci. Genome Res 11, 1275-1289

27. Martin RH, Chernos JE, Lowry RB, Pattinson HA, Barclay L, et al. (1994) Analysis of sperm chromosome complements from a man heterozygous for a paracentric inversion of chromosome 1. Hum Genet 93, $135-138$

28. McClintock B (1931) Cytological observations of deficiencies involving known genes, translocations and an inversion in Zea mays. Missouri Agricultural Experiment Research Bulletin 163, 1-30

29. McClintock B (1933) The association of non-homologous parts of chromosomes in the mid-prophase of meiosis in Zea mays. Z Zellforsch Mikrosk Anat 19, $191-237$

30. McRae AF, McEwan JC, Dodds KG, Wilson T, Crawford AM, et al. (2002) Linkage disequilibrium in domestic sheep. Genetics 160, 1113-1122

31. McRae AF, Bishop SC, Walling GA, Wilson D, Visscher PM (2005) Mapping of multiple quantitative trait loci for growth and carcass traits in a complex commercial sheep pedigree. Anim Sci 80, 135-142

32. Morton NE (1955) Sequential tests for the detection of linkage. Am J Hum Genet 7, 277-318

33. Nsengimana J, Baret P, Haley CS, Visscher PM (2004) Linkage disequilibrium in the domesticated pig. Genetics 166, 1395-1404

34. Park C, Russ I, Da Y, Lewin HA (1995) Genetic mapping of F13A to BTA23 by sperm typing: Difference in recombination rate between bulls in the DYA-PRL interval. Genomics 27, 113-118

35. Park C, Frank MT, Lewin HA (1999) Fine-mapping of a region of variation in recombination rate on BTA23 to the D23S22-D23S23 interval using sperm typing and meiotic breakpoint analysis. Genomics 59, 143-149

36. Pinton A, Ducos A, Berland HM, Séguéla A, Blanc MF, et al. (1997) A new Robertsonian translocation in the Holstein breed. Genet Sel Evol 29, 523-526

37. Popescu CP, Boscher J (1986) A new reciprocal translocation in a hypoprolific boar. Genet Sel Evol 18, $123-130$

38. Ramos PS, Bitgood JJ, De Ponce Leon A (1999) Novel chromosomal translocation in chicken uncovered by double colour FISH. Anim Biotechnol 10, 119-122

39. Rohrer GA, Alexander LJ, Hu Z, Smith TP, Keele JW, et al. (1996) A comprehensive map of the porcine genome. Genome Res 6, 371-391

40. Schmid M, Nanda I, Guttenbach M, Steinlein C, Hoehn $\mathrm{H}$, et al. (2000) First report on chicken genes and chromosomes 2000. Cytogenet Cell Genet 90, $169-218$

41. Sinsheimer JS, Blangero J, Lange K $(2000)$ Gametecompetition models. Am J Hum Genet 66, 1168-1172

42. Slate J, van Stijn TC, Anderson RM, McEwan KM, Maqbool NJ, et al. (2002) A deer (subfamily Cervinae) genetic linkage map and the evolution of ruminant genomes. Genetics 160, 1587-1597 